\title{
Nanodentures and Mechanical Electrodynamics: Three-Dimensional Relative Orientation of Plasmonic Nanoarches from Absorption Spectra
}

\author{
Robert C. Boutelle, Yi Gao, Chris Arntsen, and Daniel Neuhauser* \\ Department of Chemistry and Biochemistry, University of California, Los Angeles (UCLA), Los Angeles, California 90095-1569, \\ United States
}

\section{Supporting Information}

\begin{abstract}
We propose a general theme, labeled mechanical electrodynamics, where the relative three-dimensional (3D) orientation of particles with nontrivial geometries is tracked based on the details of the absorption spectrum beyond a onedimensional (1-D) distance dependence. Specifically, we simulate absorption spectra of a subwavelength denture-like nanostructure with freely moving parts. The nanodentures are made of two gold nanoarches that either open and close or rotate about a single arch base (hinge rotation). We show how

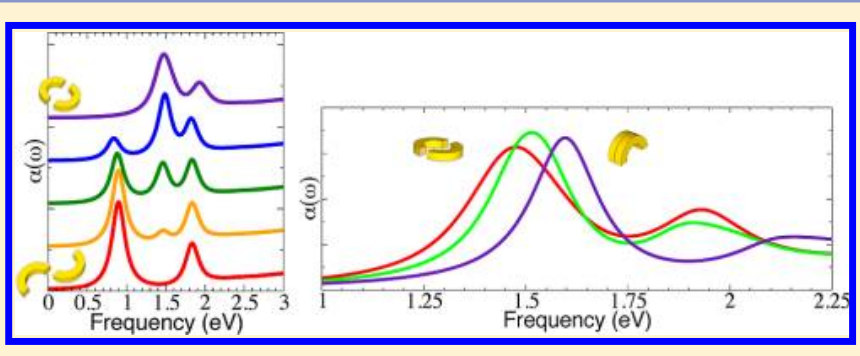
the absorption spectrum for the nanodentures changes depending on orientation and position. There is a $\sim 0.1-0.2 \mathrm{eV}$ shift in absorbance peak frequencies as the denture closes, corresponding to an increased coupling between the two gold arches, while a hinge rotation results in a depletion of one absorbance peak $(1.48 \mathrm{eV})$ with the simultaneous emergence of a new absorbance peak at lower frequencies $(0.88 \mathrm{eV})$. The unique spectral signature of each position and orientation of the nanodentures points to a variety of applications. One will be experimentally tracking and measuring orientation and position of plasmonic-coupled nanoparticles using simple methods such as UV-vis or IR spectral analysis. Additionally, the denture structure will tune in and out of different plasmon resonance frequencies, or turn "on and off," depending on its orientation. The simulations were performed efficiently by the recent near-field (NF) approach, which is a time-dependent Poisson algorithm that shares a lot of the machinery of full-fledged Maxwell equations but allows for much larger time steps and therefore can treat large systems.
\end{abstract}

\section{INTRODUCTION}

Plasmonics on the nanoscale shows promise for chemical and biomedical sensing, ${ }^{1,2}$ cancer treatment, ${ }^{3-5}$ and optoelectronic devices. ${ }^{6-9}$ With control over composition, size, and shape of material, absorption in different materials can be tuned from the IR to the UV-vis. ${ }^{10-12}$ There has therefore been much effort to characterize and understand these plasmonic phenomena. ${ }^{13-20}$ However, most systems are static, which gives rise to a single plasmonic spectrum. An alternative method of plasmonic tuning is to allow mobility and coordination of the nanoparticles. Thus, a system's plasmonic response depends on the orientation and distance of different plasmonic particles. We label this dependence mechanical electrodynamics.

Previous work on the electrodynamic response of dynamic systems has been limited. Most studies use the symmetry of the system to simplify the model, such as spheres ${ }^{16,21,22}$ or rods ${ }^{23}$ at different distances from one another. For example, colloid dimers shift their plasmonic resonance as they get closer or further away from one another. ${ }^{21,22}$ Dimers have therefore been used for tracking purposes, ${ }^{24}$ but most studies are limited to one-dimensional movement, which limits the types of response that is collected. The less symmetry the nanoparticles have, the more variation is available in the spectral signature. With a more asymmetric system, such as the two nanoarches studied here, there are more degrees of coordination, which results, as shown below, in multidimensional spectral signatures.

Predicting the spectra of these less symmetric systems increases computational time. With the recently developed near-field (NF) method, ${ }^{19,25}$ computations are comparatively efficient. NF captures the electrodynamics of nanostructures at subwavelength scales. Below about a quarter of a wavelength, retardation effects can be ignored. ${ }^{25}$ This simplifies the treatment as the components of normal electric fields are neglected. Therefore, the Poisson equation is used since the time scale is not related to the inverse of the velocity of light, so a large time step can be used.

Here we use the NF approach to model the electrodynamics of a gold nanodenture structure, a system consisting of two gold nanoarches that open and close in a denture-like fashion or rotate about a hinge point (Figure 1). The mechanical electrodynamics of the system manifest as spectral shifts due to angle changes. Simultaneously, the spatial electric field was studied to help understand the plasmon coupling mechanism. The results show that there is rich information on $3 \mathrm{D}$

Received: December 16, 2012

Revised: April 10, 2013

Published: April 15, 2013 


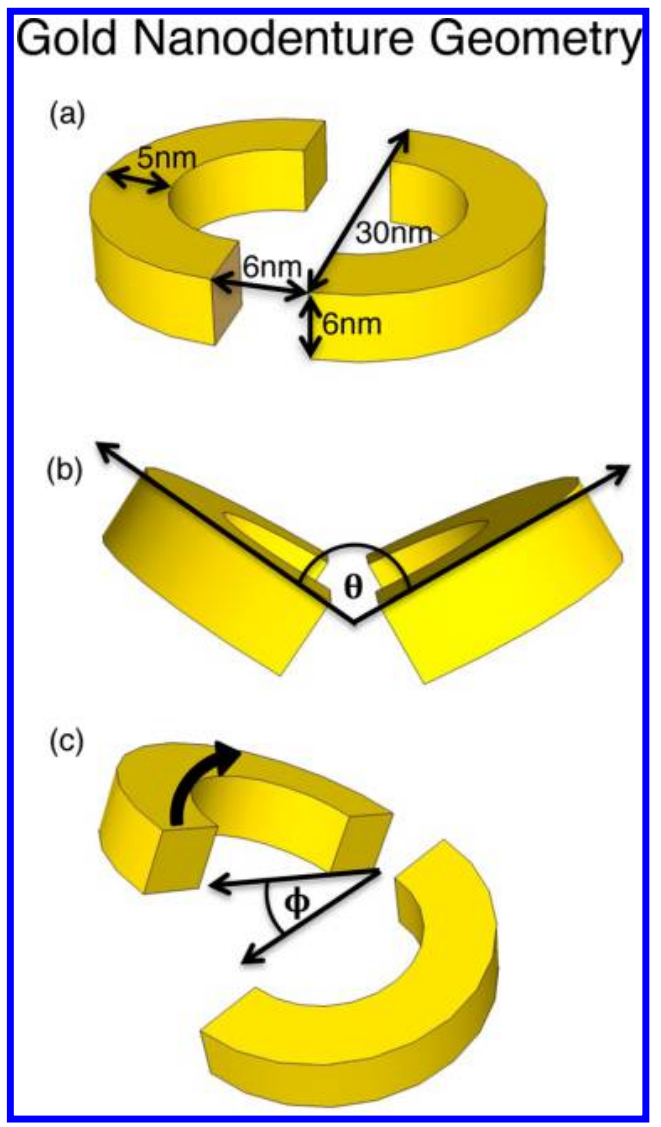

Figure 1. Model of the gold nanodenture system with (a) "flat" geometry, (b) closing mechanical motion, and (c) hinge rotation mechanical motion.

orientation in the spectral signatures of systems that are nonsymmetric.

The paper is organized as follows. section II briefly reviews $\mathrm{NF}$, the results of the simulations are presented in section III, and discussions and conclusions follow in section IV.

\section{METHOD}

$\mathrm{NF}$ is useful when the nanostructures are much smaller than the optical wavelength, in that retardation effects can be neglected. The longitudinal component of electric field then dominates so that the field is the gradient of an instantaneous scalar potential,

$$
\mathbf{E}=-\nabla \phi
$$

and fulfills the Poisson equation. The main advantage of NF is that since the method is not related to the velocity of light, the time step used for the evolution of electric field can be as high as a few atomic units, hundreds of times larger than that required in the Yee-type Maxwell finite-difference time-domain (FDTD) approach for subnanometer scales. ${ }^{26}$

NF is essentially the time-dependent version of the frequency-dependent Poisson algorithm, where one solves

$$
\nabla \cdot(\varepsilon(\mathbf{r}, \omega) \tilde{\mathbf{E}}(\mathbf{r}, \omega))=0
$$

where $\varepsilon(\mathbf{r}, \omega)$ is the permittivity of the material; $\tilde{\mathbf{E}}(\mathbf{r}, \omega)$ is the total electric field, made of the local longitudinal component and an external one. The difference from the usual Poisson algorithm is that NF is a time-domain method, and therefore all frequencies are solved for at once. In that respect, NF is similar to the FDTD algorithm, although it uses much larger time steps. In the time-domain, the electric field is

$$
\mathbf{E}(\mathbf{r}, t)=-\nabla \phi(\mathbf{r}, t)+\mathbf{E}_{\mathrm{ext}}(\mathbf{r}, t) \delta(t)
$$

As in most FDTD descriptions, NF assumes that the dielectric permittivity of a metal is represented as a sum of Drude oscillators,

$$
\varepsilon(\mathbf{r}, \omega)=\varepsilon_{\infty}(\mathbf{r})+\varepsilon_{0} \sum_{j=1}^{N} \frac{\beta_{j}(\mathbf{r})}{\bar{\omega}_{j}^{2}(\mathbf{r})-i \alpha_{j}(\mathbf{r}) \omega-\omega^{2}}
$$

Here, $\varepsilon_{\infty}(\mathbf{r})$ is the material-dependent frequency-independent term. For metals, we can assume $\varepsilon_{\infty}(\mathbf{r})=\varepsilon_{0}$. The materialdependent Drude parameters $\alpha_{j}(\mathbf{r}), \beta_{j}(\mathbf{r})$, and $\bar{\omega}_{j}(\mathbf{r})$ are realvalued and we typically apply up to $N=9$ oscillators to fit the permittivity over a wide frequency range, $0.6-6.7 \mathrm{eV}$ for gold. ${ }^{19}$

To solve eq 1 in the time domain, the FDTD-type treatment in NF develops as follows. A metallic polarization and current density is defined for each for the Drude oscillators above and is evolved as $(j=1, \ldots, N)$

$$
\frac{\partial \mathbf{P}_{j}(r, t)}{\partial t}=\mathbf{J}_{j}(\mathbf{r}, t)
$$

and

$$
\frac{\partial \mathbf{J}_{j}(\mathbf{r}, t)}{\partial t}=-\alpha_{j}(\mathbf{r}) \mathbf{J}_{j}(\mathbf{r}, t)-\bar{\omega}_{j}^{2}(\mathbf{r}) \mathbf{P}_{j}(\mathbf{r}, t)+\varepsilon_{0} \beta_{j}(\mathbf{r}) \mathbf{E}(\mathbf{r}, t)
$$

Equations 3 and 4 are propagated by the leapfrog algorithm

$$
\mathbf{P}_{\mathrm{j}}(\mathbf{r}, t+d t)=\mathbf{P}_{\mathrm{j}}(\mathbf{r}, t)+\mathrm{d} t \mathbf{J}_{\mathrm{j}}\left(\mathbf{r}, t+\frac{\mathrm{d} t}{2}\right)
$$

and

$$
\begin{aligned}
\mathbf{J}_{j}\left(\mathbf{r}, t+\frac{\mathrm{d} t}{2}\right)= & \frac{1-\frac{\alpha_{j}(\mathbf{r})}{2} \mathrm{~d} t}{1+\frac{\alpha_{j}(\mathbf{r})}{2} \mathrm{~d} t} \mathbf{J}_{j}\left(\mathbf{r}, t-\frac{\mathrm{d} t}{2}\right) \\
& -\frac{\mathrm{d} t}{1+\frac{\alpha_{j}(\mathbf{r})}{2} \mathrm{~d} t}\left(\bar{\omega}_{j}^{2}(\mathbf{r}) \mathbf{P}_{j}(\mathbf{r}, t)\right. \\
& \left.-\varepsilon_{0} \beta_{j}(r) \mathbf{E}(\mathbf{r}, t)\right)
\end{aligned}
$$

To close these equations, we need the electric field. In FDTD, the electric field is propagated as an independent variable, while in NF it is obtained from the overall potential, which is calculated in turn, at any time, from the polarization. Specifically, for purely metallic systems and a uniform initial external pulse, the electric fields is

$$
\mathbf{E}(\mathbf{r}, t=n \mathrm{~d} t)=\left\{\begin{array}{cc}
-\nabla \varphi(\mathbf{r}, t) & n>0 \\
\frac{1}{\mathrm{~d} t} \mathbf{E}_{\mathrm{ext}} & n=0
\end{array}\right.
$$

where the initial delta function in eq 2 is obtained (for purely metallic systems; see ref 25 for the extension to dielectrics) as

$$
-\nabla^{2} \varphi(\mathbf{r}, t)=\frac{\rho(\mathbf{r}, t)}{\varepsilon_{0}}
$$

where we defined a metallic charge density as

$$
\rho=-\nabla \cdot \mathbf{P}(\mathbf{r}, t)
$$


from the metallic polarization

$$
\mathbf{P}=\sum_{j} \mathbf{P}_{j}
$$

The initial values for the propagation are

$$
\mathbf{P}_{j}(r, t=0)=\mathbf{J}_{j}\left(r, t=-\frac{\mathrm{d} t}{2}\right)=0
$$

To summarize this section: for purely metallic systems, we start the simulation at $t=0$ with a uniform electric field and no polarizations and currents, then propagate eqs 5 and 6 , and at any time, step solve eq 7 by convolution.

The results are then Fourier transformed to yield $\boldsymbol{P}(\mathbf{r}, \omega)$, and the absorption spectrum is obtained from the absorption strength, defined as

$$
\alpha(\omega)=\frac{4 \pi \omega}{c\left|E_{\text {ext }}\right|^{2}} \int \operatorname{Im} \mathbf{P}(\mathbf{r}, \omega) \cdot E_{\text {ext }} \mathrm{d}^{3} \mathbf{r}
$$

where $P(\mathbf{r}, \omega)$ is the frequency-dependent total polarization. In practice, unpolarized absorbance spectra were calculated by summing the trace of $\alpha(\omega)$ with $x, y$, and $z$ polarized induced fields.

\section{RESULTS AND DISCUSSION}

A gold nanodenture structure was studied with dimensions shown in Figure 1. Our absorbance calculations were benchmarked for the open denture (Flat or $\theta=180^{\circ}$ ), halfway open $\left(\theta=90^{\circ}\right)$, and closed $\left(\theta=0^{\circ}\right)$ (see also Supporting Information, Figure 1). Two hundred mesh grid points in each dimension were necessary for satisfactory convergence with the halfway open and closed geometry, while 300 mesh grid points were necessary for the open denture geometry. The structure was then tested in two different cases: denture closure (Figure 1b) and hinge rotation (Figure 1c). For denture closure, the angle $\theta$, ranging from $180^{\circ}$ to $0^{\circ}$ in $45^{\circ}$ increments, was generated from the nanoarches rotating out of the plane toward one another while connected at both bases at the spring line (in architecture, the spring line is the point at which the arch first begins to curve to create the arch structure). With hinge rotation, the angle $\varphi$, varying between $0^{\circ}$ to $180^{\circ}$ in $45^{\circ}$ increments, was generated from the rotation of the nanoarches connected at a single base at the spring line. The spectra were monitored as shifts in absorbance peaks with the change in geometry. Experimentally, this is the equivalent of a switchable polymer linker connecting the bases of the nanoarches and controlling the mechanics of the nanodenture system.

Initially two characteristic absorbance frequency peaks are seen from a single gold arch found at $1.37 \mathrm{eV}$ and $1.97 \mathrm{eV}$ (Figure 2a). However, when another gold arch is introduced in the "flat" or $\theta=180^{\circ}$ denture position, the lower frequency peak is blue-shifted by $\sim 0.11 \mathrm{eV}$, while the higher frequency peak remains virtually unchanged. This is due to the newly introduced plasmon coupling between the two nanoarches.

As the dentures close (Figure $1 \mathrm{~b}$ ), the absorbance frequency peaks are blue-shifted by $0.1-0.2 \mathrm{eV}(1.48 \mathrm{eV}$ to $1.60 \mathrm{eV}$ for the low frequency peak and $1.93 \mathrm{eV}$ to $2.16 \mathrm{eV}$ for the high frequency peak) (Figure 2b). This shift is explained by a stronger coupling between the two arches as the dentures close compared to the flat geometry. With more surface area and a smaller distance between the arches, there is a stronger coupling, which causes the peak shifts in the spectrum.

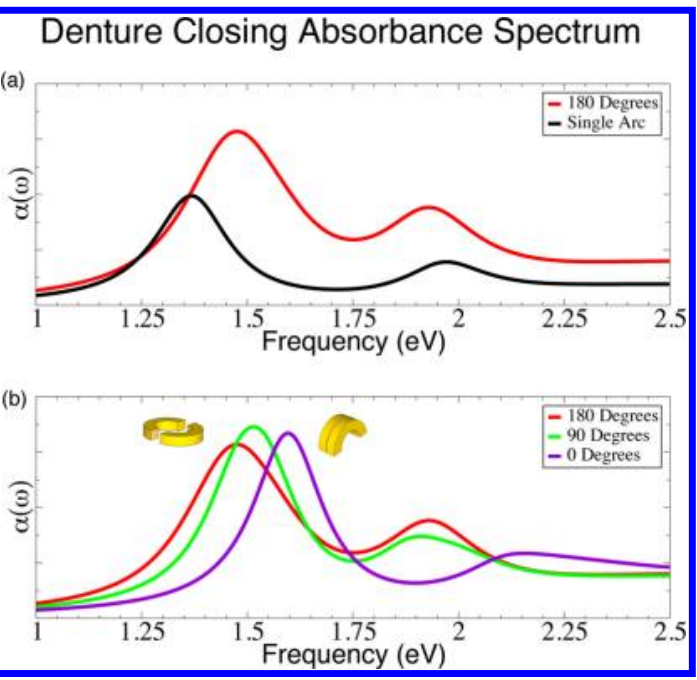

Figure 2. (a) The calculated absorbance spectra of a single gold nanoarch and two gold nanoarches in a denture-like geometry. The lower frequency peak blue shifts due to coupling between the two arches. (b) As the arches close, the frequency of absorption shifts higher. This is due to increased coupling between the two arches. Intermediate angles shown are found in Supporting Information, Figure 2.

Interestingly, the initial $180^{\circ}$ to $90^{\circ}$ angle change does not shift the frequency peaks drastically compared with the last $90^{\circ}$ to $0^{\circ}$. This is due to the limited range of the plasmon coupling in the arches. The last $90^{\circ}$ to $0^{\circ}$ angles puts the surfaces of the two arches much closer to one another than the $180^{\circ}$ to $90^{\circ}$ angles initially do. Thus, there is a stronger interaction between the two nanoarches between $90^{\circ}$ to $0^{\circ}$ angle.

For the hinge rotation case (Figure 1c), a drastically different effect is seen. Instead of a gradual shift in absorbance frequency peaks, there is a disappearance of a dominant absorbance peak and the emergence of another (Figure 3 ). As the arch rotates about one of the other arch's base, the absorbance frequency

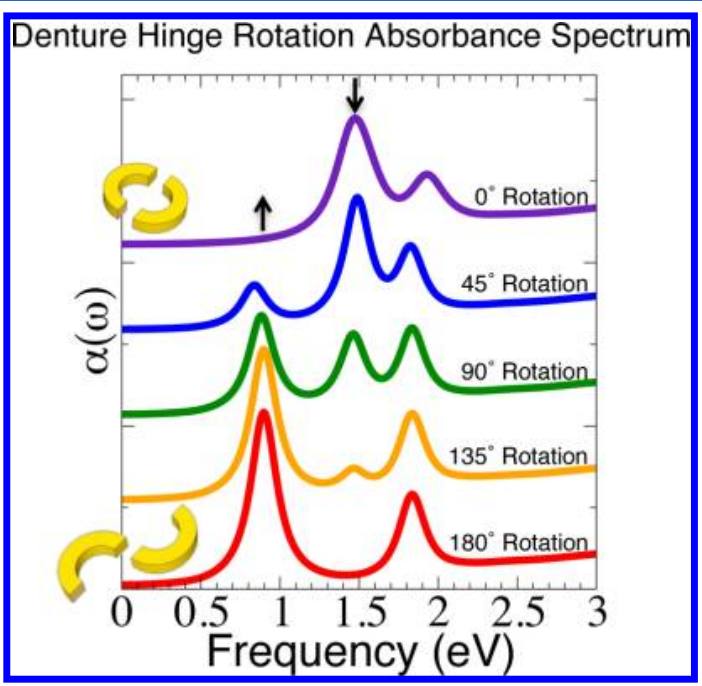

Figure 3. Absorption spectrum of a gold nanodenture system as the arches rotate about a single arch base at the spring line. As the arches rotate, the frequency of absorbance turns "on and off" as a major peak disappears while another emerges (indicated by the arrows). This trend is due to a decreased coupling of the nanoarches at the spring line. 
peak at $1.48 \mathrm{eV}$ decreases while the absorbance frequency peak at $0.88 \mathrm{eV}$ starts to emerge. By the time the nanoarch has rotated $180^{\circ}$, the first frequency peak $(1.48 \mathrm{eV})$ has fully depleted, while the new frequency peak $(0.88 \mathrm{eV})$ has fully emerged. This happens in a fairly linear fashion, illustrated by the nearly equal intensity levels of both peaks at the $90^{\circ}$ rotation. The frequency shift is due to a decoupling of the two nanoarches as an arch rotates away from the other arch. It is important to note the large frequency difference of $0.6 \mathrm{eV}$ from the two peaks. This will be useful for devices that need to be tuned in and out of a plasmonic frequency, or "on and off."

Additionally, note how different the two methods of plasmonic tuning are due to different mechanical electrodynamic properties of the system. This illustrates the versatility in which less symmetric systems can be tuned. Unlike the dual nanocolloid system, gold nanoarches have a multifaceted way of tuning themselves with different mechanical movements giving potentially very different spectral results. Furthermore, each orientation gives a unique result. This is important for tracking orientation and position via far-field methods such as UV-vis and IR absorption, as each orientation and position has a unique spectral signature. This also gives rise to avenues of mechanically tuning and detuning plasmons through rearranging the nanostructure's orientation.

To help analyze the characteristic effects of the mechanical electrodynamics, the spatial electric field was simulated by NF for the gold nanodenture structure (Figure 4). For ease of

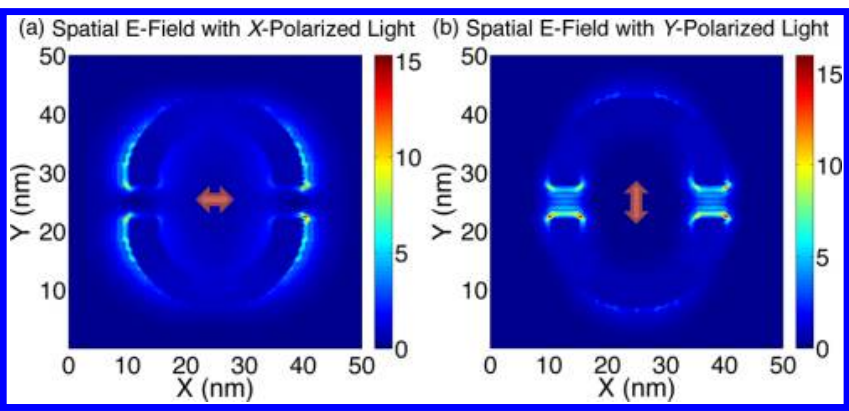

Figure 4. Spatial energy distribution of the gold nanodenture system for (a) $x$-polarized light and (b) $y$-polarized light. The local hotspots can be seen near the hinge points of the nanodenture system. The double arrows illustrate the direction of polarization.

visualization, the flat (or $0^{\circ}$ ) nanostructure was chosen. The electric field response was visualized in the $z$-plane that bisects the gold nanodentures. Inside the material, the electric field response is roughly zero due to no net change in electric field. Thus the $z$-polarized light is not included in the figures. For $x$ polarized electric fields, the response is greatest along the outside rim near the spring line of the nanoarches. For $y$ polarized electric fields, the electric field is greatest between the two arches at the bases, though is not insubstantial at the tip of the arcs. This spatial information helps explain why the frequency of absorption peaks shift.

The $x$-polarized and $y$-polarized electric field images illustrate the initial coupling between the two nanoarches and thus explain the initial frequency peak shift as this coupling is initiated. It also helps explain the characteristic effect of both the denture closing case and the hinge rotation case. As the nanodenture starts to rearrange, the coupling starts to change. Since the main source of coupling is initially at the hinge point, it is not surprising that the spectrum changes as the orientation around the hinge point changes, either from the dentures closing or a hinge rotation.

With the spectra of systems and identifying coupling regions, or hot spots, it is clear that these less symmetric systems have complex properties that need to be explored.

\section{CONCLUSION}

With less symmetric nanostructured systems, there is more complexity in orientation and position. This leads to interesting coupling dynamics with the plasmons, and the NF-predicted spectrum for the gold nanodentures illustrates this phenomenon well. Depending on how the system is changed, either denture closing or hinge rotation, the spectrum changes in drastically different ways. There is a unique $\sim 0.1-0.2 \mathrm{eV}$ frequency peak shift for the two major peaks of the gold nanoarches as the nanodenture closes. On the other hand, there is a peak depletion at $1.48 \mathrm{eV}$ and a peak emergence at $0.88 \mathrm{eV}$ for a hinge rotation.

The versatility in the mechanical electrodynamics leads to potentially interesting applications. Due to the nature of the shifts and changes in intensity of the peaks caused by the mechanical electrodynamics, applications in tracking via far field methods such as UV-vis and IR absorption could be envisioned. Additionally, if the orientation could be controlled via a linking molecule or polymer, the plasmon could be switched in and out of resonance with ease. This could be useful for plasmonic devices that require an "on and off" state.

In future studies, it would be interesting to extend the NF method to other frequency regimes. It would also be interesting to further study other less symmetric systems or a completely asymmetric system to see what sort of mechanical electrodynamics could be exploited for experimental purposes and to search for optimal structures in which the spectrum changes even more drastically and uniquely depending on the relative orientation.

\section{ASSOCIATED CONTENT}

\section{S Supporting Information}

Extra figures concerning convergence of the absorption calculations and intermediate geometries for Figure 2 can be found in the Supporting Information. This material is available free of charge via the Internet at http://pubs.acs.org.

\section{AUTHOR INFORMATION}

\section{Corresponding Author}

*E-mail: dxn@chem.ucla.edu. Telephone: 310-206-1274. Address: 607 Charles E. Young Drive East, Los Angeles, CA 90095-1569.

\section{Notes}

The authors declare no competing financial interest.

\section{ACKNOWLEDGMENTS}

We are grateful for support from the National Science Foundation (NSF), Grant CHE-1112500, and to Louis Bouchard for insightful discussions.

\section{REFERENCES}

(1) Matsubara, K.; Kawata, S.; Minami, S. Optical Chemical Sensor Based on Surface Plasmon Measurement. Appl. Opt. 1988, 27 (6), $1160-1163$.

(2) Khlebtsov, N. G.; Dykman, L. A. Optical Properties and Biomedical Applications of Plasmonic Nanoparticles. J. Quant. Spectrosc. Radiat. Transfer 2010, 111 (1), 1-35. 
(3) Chen, J.; Glaus, C.; Laforest, R.; Zhang, Q.; Yang, M.; Gidding, M.; Welch, M. J.; Xia, Y. Gold Nanocages as Photothermal Transducers for Cancer Treatment. Small 2010, 6 (7), 811-817.

(4) Hühn, D.; Govorov, A.; Gil, P. R.; Parak, W. J. Photostimulated $\mathrm{Au}$ Nanoheaters in Polymer and Biological Media: Characterization of Mechanical Destruction and Boiling. Adv. Funct. Mater. 2012, 22 (2), 294-303.

(5) Richardson, H. H.; Carlson, M. T.; Tandler, P. J.; Hernandez, P.; Govorov, A. O. Experimental and Theoretical Studies of Light-to-Heat Conversion and Collective Heating Effects in Metal Nanoparticle Solutions. Nano Lett. 2009, 9 (3), 1139-1146.

(6) Garcia, G.; Buonsanti, R; Runnerstrom, E. L.; Mendelsberg, R. J.; Llordes, A.; Anders, A.; Richardson, T. J.; Milliron, D. J. Dynamically Modulating the Surface Plasmon Resonance of Doped Semiconductor Nanocrystals. Nano Lett. 2011, 11 (10), 4415-4420.

(7) Atwater, H. A.; Polman, A. Plasmonics for Improved Photovoltaic Devices. Nat. Mater. 2010, 9 (3), 205-213.

(8) Chang, D. E.; Sørensen, A. S.; Demler, E. A.; Lukin, M. D.; Single-Photon, A. Transistor Using Nanoscale Surface Plasmons. Nat. Phys. 2007, 3 (11), 807-812.

(9) Neuhauser, D.; Lopata, K. Molecular Nanopolaritonics: Cross Manipulation of Near-Field Plasmons and MoleculesJ. I. Theory and Application to Junction Control. J. Chem. Phys. 2007, 127, 154715.

(10) Biteen, J. S.; Lewis, N. S.; Atwater, H. A.; Mertens, H.; Polman, A. Spectral Tuning of Plasmon-Enhanced Silicon Quantum Dot Luminescence. Appl. Phys. Lett. 2006, 88 (13), 131109-1-131109-3.

(11) Halas, N. Playing with Plasmons: Tuning the Optical Resonant Properties of Metallic Nanoshells. MRS Bull. 2005, 30 (05), 362-367.

(12) Yelk, J.; Sukharev, M.; Seideman, T. Optimal Design of Nanoplasmonic Materials Using Genetic Algorithms as a MultiParameter Optimization Tool. arXiv.org, e-Print Arch, Phys. Opt. 2008, No. arXiv:0802.2899.

(13) Esteban, R.; Borisov, A. G.; Nordlander, P.; Aizpurua, J. Bridging Quantum and Classical Plasmonics with a Quantum-Corrected Model. Nat. Commun. 2012, 3, 825.

(14) Halas, N. J.; Lal, S.; Chang, W.-S.; Link, S.; Nordlander, P. Plasmons in Strongly Coupled Metallic Nanostructures. Chem. Rev. 2011, 111 (6), 3913-3961.

(15) Morton, S. M.; Silverstein, D. W.; Jensen, L. Theoretical Studies of Plasmonics Using Electronic Structure Methods. Chem. Rev. 2011, 111 (6), 3962-3994.

(16) McMahon, J.; Henry, A.-I.; Wustholz, K.; Natan, M.; Freeman, R.; Van Duyne, R.; Schatz, G. Gold Nanoparticle Dimer Plasmonics: Finite Element Method Calculations of the Electromagnetic Enhancement to Surface-Enhanced Raman Spectroscopy. Anal. Bioanal. Chem. 2009, 394 (7), 1819-1825.

(17) Arntsen, C.; Lopata, K.; Wall, M. R.; Bartell, L.; Neuhauser, D. Modeling Molecular Effects on Plasmon Transport: Silver Nanoparticles with Tartrazine. J. Chem. Phys. 2011, 134 (8), 084101-1084101-7.

(18) Lopata, K.; Neuhauser, D. Nonlinear Nanopolaritonics: FiniteDifference Time-Domain Maxwell-Schrödinger Simulation of Molecule-Assisted Plasmon Transfer. J. Chem. Phys. 2009, 131, 014701.

(19) Coomar, A.; Arntsen, C.; Lopata, K. A.; Pistinner, S.; Neuhauser, D. Near-Field: A Finite-Difference Time-Dependent Method for Simulation of Electrodynamics on Small Scales. J. Chem. Phys. 2011, $135,084121$.

(20) Lopata, K.; Neuhauser, D.; Baer, R. Curve Crossing and Negative Refraction in Simulations of Near-Field Coupled Metallic Nanoparticle Arrays. J. Chem. Phys. 2007, 127, 154714.

(21) Nordlander, P.; Oubre, C.; Prodan, E.; Li, K.; Stockman, M. I. Plasmon Hybridization in Nanoparticle Dimers. Nano Lett. 2004, 4 (5), 899-903.

(22) Yang, S.-C.; Kobori, H.; He, C.-L.; Lin, M.-H.; Chen, H.-Y.; Li, C.; Kanehara, M.; Teranishi, T.; Gwo, S. Plasmon Hybridization in Individual Gold Nanocrystal Dimers: Direct Observation of Bright and Dark Modes. Nano Lett. 2010, 10 (2), 632-637.
(23) Funston, A. M.; Novo, C.; Davis, T. J.; Mulvaney, P. Plasmon Coupling of Gold Nanorods at Short Distances and in Different Geometries. Nano Lett. 2009, 9 (4), 1651-1658.

(24) Rong, G.; Wang, H.; Skewis, L. R.; Reinhard, B. M. Resolving Sub-diffraction Limit Encounters in Nanoparticle Tracking Using Live Cell Plasmon Coupling Microscopy. Nano Lett. 2008, 8 (10), 33863393.

(25) Li, S.; Gao, Y.; Neuhauser, D. Near-Field for Electrodynamics at Sub-wavelength Scales: Generalizing to an Arbitrary Number of Dielectrics. J. Chem. Phys. 2012, 136, 234104.

(26) Taflov, A.; Hagness, S. Computational Electrodynamics: The Finite-Difference Time-Domain Method, 2nd ed.; Artech House: Boston, MA, 2000. 